山्山FFRANÇAISE

$\supset$ DE

필 PEAGOGIE

\section{Revue française de pédagogie}

Recherches en éducation

157 | octobre-décembre 2006

PISA : analyses secondaires, questions et débats

théoriques et méthodologiques

\title{
MALET Régis \& BRISARD Estelle (dir.). Modernisation de l'école et contextes culturels: des politiques aux pratiques en France et en Grande-Bretagne
}

Préface d'Agnès van Zanten. Paris : L'Harmattan, 2005. - 277 p. (Éducation comparée).

Romuald Normand

\section{(Q) OpenEdition}

Édition électronique

URL : http://journals.openedition.org/rfp/483

DOI : $10.4000 /$ rfp. 483

ISSN : 2105-2913

Éditeur

ENS Éditions

Édition imprimée

Date de publication : 1 décembre 2006

Pagination : 189-191

ISBN : 978-2-7342-1075-7

ISSN : 0556-7807

Référence électronique

Romuald Normand, « MALET Régis \& BRISARD Estelle (dir.). Modernisation de l'école et contextes culturels des politiques aux pratiques en France et en Grande-Bretagne », Revue française de pédagogie [En ligne], 157 | octobre-décembre 2006, mis en ligne le 14 octobre 2010, consulté le 25 septembre 2020. URL http://journals.openedition.org/rfp/483 ; DOI : https://doi.org/10.4000/rfp.483

Ce document a été généré automatiquement le 25 septembre 2020

(c) tous droits réservés 


\section{MALET Régis \& BRISARD Estelle (dir.). Modernisation de l'école et contextes culturels : des politiques aux pratiques en France et en Grande-Bretagne}

Préface d'Agnès van Zanten. Paris : L'Harmattan, 2005. - 277 p. (Éducation comparée).

\section{Romuald Normand}

\section{RÉFÉRENCE}

MALET Régis \& BRISARD Estelle (dir.). Modernisation de l'école et contextes culturels : des politiques aux pratiques en France et en Grande-Bretagne / préface d'Agnès van Zanten. Paris : L’Harmattan, 2005. - 277 p. (Éducation comparée).

En France, les comparaisons internationales demeurent un exercice marginal alors qu'elles constituent un véritable champ de recherche outre-Manche et qu'elles s'appuient sur des revues scientifiques de qualité. C'est le mérite du livre de Régis Malet et d'Estelle Brisard de nous rappeler que nul n'est prophète en son pays et que la méthode comparative est aussi, comme l'écrivait Émile Durkheim, au fondement de l'administration de la preuve. L'ouvrage prend comme fil conducteur les transformations du métier d'enseignant pour interroger les effets de convergence des politiques éducatives en France et en Angleterre et les spécificités nationales ou locales, notamment les contextes culturels, qui résistent à l'emprise de la globalisation. Si les systèmes éducatifs français et anglais sont soumis à un même impératif de rationalisation, selon des exigences renforcées d'efficacité et de rentabilité, les expériences sociales des enseignants y sont très différentes. Ces différences, minutieusement analysées, montrent aussi que l'adaptation de la profession enseignante à la diversification des publics scolaires et à l'enseignement de masse 
présente des configurations spécifiques dont une approche macrosociologique ne peut à elle seule rendre compte. D'où l'intérêt d'une démarche comparative dont les principes, décrits par Marie-Pierre Moreau, relèvent de choix méthodologiques et théoriques qui accordent une place essentielle aux contextes locaux et à la dynamique des acteurs mais respectent aussi un principe de précaution en termes d'équivalence linguistique ou conceptuelle.

2 Une première série d'articles entreprend de saisir les dynamiques transnationales qui, en dépit du développement d'un champ de références internationales et des transformations de l'État éducateur, interagissent avec des traditions locales, des formes d'administration scolaire, et des modalités de formation des enseignants, en opérant une médiation sociale et culturelle propre à chaque pays. Ainsi, comme l'affirme Jenny Ozga, si des politiques « nomades » tendent à définir un certain nombre d'objectifs au niveau supranational en termes de modernisation des politiques d'éducation, selon des rhétoriques et des stratégies cohérentes visant à promouvoir un agenda néo-libéral, les logiques d'appropriation de ces référentiels par les acteurs locaux témoignent de politiques "enracinées ", comme l'illustre l'exemple écossais, dans lesquelles se maintiennent des récits collectifs attentifs au maintien d'une identité nationale et à la préservation d'un bien commun. La mise en parallèle par Régis Malet de l'histoire des systèmes d'enseignement fournit une autre direction pour penser les évolutions de l'administration scolaire en Angleterre et en France. Si les deux pays semblent avoir vécu des trajectoires opposées, centralisation du curriculum et de l'évaluation dans un pays à forte tradition locale pour l'Angleterre, lois de décentralisation déléguant les compétences de l'État aux collectivités territoriales dans le cas de la France, des traits similaires peuvent être identifiés : autonomisation des établissements, mise en œuvre de régulations intermédiaires, développements de l'évaluation. Toutefois, cette convergence formelle des systèmes éducatifs masque des différences en matière de construction des savoirs scientifiques. Alors que la recherche anglaise hésitait entre un accompagnement du management scolaire proche de l'expertise et un travail critique des effets négatifs des réformes néo-libérales, l'étude du travail et de la professionnalisation des enseignants en France accompagnait la découverte du local et l'ouverture de la boîte noire de l'établissement scolaire.

3 C'est de cette tradition que se réclament indirectement les articles d'Anne Barrère et de Aziz Jellab qui livrent ici une analyse comparée des expériences du travail enseignant dans le contexte de l'enseignement secondaire français. Pour Anne Barrère, c'est davantage la façon dont les enseignants traversent les épreuves propres à leur activité professionnelle qui expliquent les différences entre les situations vécues plutôt que les caractéristiques des établissements scolaires selon qu'ils accueillent des élèves difficiles ou non. C'est pourquoi, selon le deuil qu'ils font de leur discipline, la nature de la confrontation quotidienne avec les élèves, leur accommodement aux formes de l'évaluation, et le degré de reconnaissance dont ils bénéficient, les enseignants ressentiront avec plus ou moins de force les contraintes de leur environnement. De même, comme le décrit Aziz Jellab pour les lycées professionnels, les éléments constitutifs du travail enseignant varient selon le rapport au savoir que privilégient les professeurs et le type de relations qu'ils adoptent avec leurs élèves. Alors que le profil social et professionnel des enseignants de lycées professionnels a évolué, et que les élèves entretiennent des rapports de plus en plus distanciés avec la culture ouvrière, différentes postures pédagogiques se juxtaposent selon la place accordée à 
l'accompagnement des élèves et à la transmission des savoirs. Toutefois, ces postures individuelles s'inscrivent de plus en plus dans des configurations plurielles du travail collectif qui résultent de l'émergence d'une forme plus libérale de l'investissement pédagogique autour $\mathrm{du}$ projet et d'une nouvelle distribution des tâches dans l'établissement scolaire. Marie-Christine Lefloch décrit ainsi la multiplicité des configurations qui caractérisent cette coordination de l'action à plusieurs, depuis les couples professionnels ou les triades d'enseignants rassemblés autour d'un projet jusqu'aux réseaux plus institutionnels pour lesquels les liens de proximité sont plus distendus.

4 Les formes d'organisation du travail des enseignants sont malgré tout assez différentes en France et en Angleterre. Côté français, le repli de l'enseignant sur la salle de classe et l'absence d'injonction au travail collectif témoignent d'une norme professionnelle où la revendication de l'autonomie pédagogique est corollaire de l'appartenance à une communauté sociale limitée entre pairs. Dans le système éducatif anglais, une prescription managériale beaucoup plus forte s'appuie sur différentes formes de leadership et un collectif de travail beaucoup plus structurant dans les pratiques pédagogiques. L'inscription du travail enseignant dans une éthique communautaire s'entrecroise avec une culture organisationnelle propice à la mise en œuvre d'un professionnalisme collectif soucieux d'une mise en commun à des fins d'efficacité. Ces divergences permettent de mieux comprendre la manière dont les enseignants de chaque pays s'approprient les réformes scolaires et définissent leur propre travail. À partir de plusieurs comparaisons internationales entre la France et l'Angleterre, Marilyn Osborn et Elizabeth Mc Ness ont pu démontré que la mise en œuvre des politiques éducatives faisaient l'objet de médiations sociales et culturelles importantes et qu'il était nécessaire de prendre en compte les attitudes complexes des enseignants confrontés au changement. La conception de l'étendue des responsabilités éducatives varie sensiblement, les enseignants anglais oscillant entre acceptation professionnelle $\mathrm{du}$ changement et sentiment de démoralisation face aux pressions du management, alors que les enseignants français témoignent d'un profond scepticisme et d'une lassitude beaucoup plus grande vis-à-vis des réformes. Ces différences se font ressentir jusque dans l'enseignement des mathématiques, qu'elles concernent la gestion du groupe-classe, les routines pédagogiques, l'usage du manuel scolaire, comme en témoigne l'article de Birgit Pepin. Au travers d'une ethnographie très précise inspirée du travail de Marcel Mauss, Maroussia Raveau montre que ces variations sont aussi perceptibles dans la mise en ordre des corps et les différentes postures adoptées par les élèves à l'école primaire.

5 L'ensemble de ces contributions, fort bien documentées et riche d'exemples, offre au lecteur l'occasion d'un décentrement et d'une réflexion approfondie sur les changements à l'œuvre dans la profession enseignante à l'aune des réformes éducatives propres à chaque pays. Elles montrent bien l'intrication et l'hybridation de processus complexes qui, du local au global, contribuent à un déplacement des modes d'organisation du travail et de la gestion quotidienne des établissements scolaires. Sans doute aurait-on pu attendre de la part de Régis Malet et d'Estelle Brisard une réflexion plus distanciée sur les notions de modernisation, d'organisation apprenante, de gouvernance qui sont autant éléments d'une rhétorique managériale que des concepts proprement sociologiques. De même, dans un souci de comparaison, il aurait été opportun de souligner davantage côté anglais le contexte et les effets des politiques de «shame and blame» (honte et blâme) de la profession enseignante soumise depuis deux 
décennies à la pression d'un État managérial très différent d'un modèle bureaucratique contrairement à ce qu'il est écrit dans le livre. Enfin, même si l'ouvrage ne pouvait pas tout traiter, il est dommage qu'aucun article ne décrive la transformation du rôle des corps d'inspection en Angleterre qui ont pourtant largement contribué à la mise en œuvre et à l'accompagnement des réformes dans un sens néo-libéral, tandis que leurs fonctions demeuraient plus traditionnelles en France. Mais, au delà de ses faiblesses toute relatives, le livre constitue un exercice réussi d'analyse comparée qui, par l'étendue de sa bibliographie et de ses études de cas, offre une mise en perspective intéressante et un cadre stimulant de réflexion pour l'expert comme pour le néophyte.

\section{AUTEURS}

ROMUALD NORMAND

INRP

UMR Éducation \& Politiques 